\title{
Randomised controlled trial of transanal endoscopic microsurgery versus endoscopic mucosal resection for large rectal adenomas (TREND Study)
}

\author{
Renée M Barendse, ${ }_{1}^{1}$ Gijsbert D Musters, ${ }^{1}$ Eelco J R de Graaf, ${ }^{2}$ \\ Frank J C van den Broek, ${ }^{3}$ Esther C J Consten, ${ }^{4}$ Pascal G Doornebosch, ${ }^{2}$ \\ James C Hardwick, ${ }_{1}^{5}$ Ignace H J T de Hingh ${ }_{1}^{6}$ Chrisiaan Hoff, ${ }^{7}$ Jeroen M Jansen, ${ }_{1}^{8}$ \\ A W Marc van Milligen de Wit, ${ }^{9}$ George P van der Schelling, ${ }^{10}$ Erik J Schoon, ${ }^{11}$ \\ Matthijs P Schwartz, ${ }_{12}$ Bas L A M Weusten, ${ }^{13}$ Marcel G Dijkgraaf, ${ }^{14}$ Paul Fockens, ${ }^{15}$ \\ Willem A Bemelman, ${ }^{1}$ Evelien Dekker, ${ }^{15}$ on behalf of the TREND Study group
}

- Additional material is published online only. To view please visit the journal online (http://dx.doi.org/10.1136/ gutjnl-2016-313101).

For numbered affiliations see end of article.

Correspondence to Renée M Barendse, Department of Surgery, Academic Medical Centre, Amsterdam 1105 AZ, Netherlands; r.m.barendse@ amc.uva.nl

Received 26 September 2016 Revised 2 May 2017 Accepted 3 May 2017 Published Online First 28 June 2017
Check for updates

To cite: Barendse RM Musters GD, de Graaf EJR, et al. Gut 2018:67:837-846.

\section{ABSTRACT}

Objective Non-randomised studies suggest that endoscopic mucosal resection (EMR) is equally effective in removing large rectal adenomas as transanal endoscopic microsurgery (TEM), but EMR might be more cost-effective and safer. This trial compares the clinical outcome and cost-effectiveness of TEM and EMR for large rectal adenomas.

Design Patients with rectal adenomas $\geq 3 \mathrm{~cm}$, without malignant features, were randomised $(1: 1)$ to EMR or TEM, allowing endoscopic removal of residual adenoma at 3 months. Unexpected malignancies were excluded postrandomisation. Primary outcomes were recurrence within 24 months (aiming to demonstrate non-inferiority of EMR, upper limit 10\%) and the number of recurrencefree days alive and out of hospital.

Results Two hundred and four patients were treated in 18 university and community hospitals. Twenty-seven (13\%) had unexpected cancer and were excluded from further analysis. Overall recurrence rates were 15\% after EMR and 11\% after TEM; statistical non-inferiority was not reached. The numbers of recurrence-free days alive and out of hospital were similar (EMR 609 \pm 209 , TEM $652 \pm 188, p=0.16)$. Complications occurred in $18 \%$ (EMR) versus $26 \%$ (TEM) $(p=0.23)$, with major complications occurring in 1\% (EMR) versus $8 \%$ (TEM) $(p=0.064)$. Quality-adjusted life years were equal in both groups. EMR was approximately $€ 3000$ cheaper and therefore more cost-effective.

Conclusion Under the statistical assumptions of this study, non-inferiority of EMR could not be demonstrated. However, EMR may have potential as the primary method of choice due to a tendency of lower complication rates and a better cost-effectiveness ratio. The high rate of unexpected cancers should be dealt with in further studies.

\section{INTRODUCTION}

Rectal cancer is a common disease in the Netherlands with approximately 4350 new cases annually. ${ }^{1}$ The incidence of rectal cancer is increased with high age, male sex and obesity, without ethnic preference. ${ }^{2-4}$ In the pathogenesis, premalignant intraepithelial

\section{Significance of this study}

What is already known on this subject?

- After single intervention, endoscopic mucosal resection (EMR) for large rectal adenomas appears to be less effective but safer than transanal endoscopic microsurgery (TEM). The effectiveness becomes similar, when a second resection of adenoma remnants is allowed. However, the added morbidity of re-treatment is unknown.

- The current evidence lacks comparative studies and is insufficient to make firm recommendations.

What are the new findings?

- Recurrence occurred more often than expected after both EMR and TEM. Non-inferiority of EMR could not be demonstrated, partly due to a small absolute difference in recurrence rates.

- EMR appears to be the more cost-effective technique.

- Both interventions were associated with significant postprocedural morbidity.

Complications of TEM tended to be major more often.

- Another important finding was a rather high rate of unexpected malignancies in rectal adenomas that were endoscopically diagnosed as benign.

neoplasia in a rectal adenoma precedes the occurrence of invasive rectal cancer. ${ }^{56}$ Early endoscopic detection and removal of rectal adenomas prevents the development of rectal cancer and decreases rectal cancer related mortality. ${ }^{78}$ It can be expected that due to the worldwide increasing implementation of colorectal cancer screening programmes, the detection of adenomas will increase. For small adenomas, standard endoscopic therapies like simple snare polypectomy or one-step endoscopic mucosal resection (EMR) will suffice. When 
Significance of this study

How might it impact on clinical practice in the foreseeable future?

- Data of this trial reflect daily practice in the Netherlands and Belgium, where gastroenterologists and surgeons with a specific dedication perform EMR and TEM. They have much lower case-volumes compared with the few expert centres dominating the current literature.

- EMR should be considered as a potential treatment alternative. EMR deserves further attention and development in expert centres, in order to coexist besides TEM, or become the better alternative, in the treatment portfolio for large rectal adenomas.

rectal adenomas are large, however, these techniques will be inadequate.

Surgical treatment of large rectal adenomas has advanced rapidly; local resection techniques and staging techniques (endorectal ultrasonography in particular) have been improved. In the past, rectal adenomas that were too large for endoscopic removal were referred for transanal resection or even total mesorectal excision (TME). The former technique was associated with a high incidence of recurrences, whereas the latter had a high operative morbidity and long-term functional consequences. ${ }^{910}$ In 1984, transanal endoscopic microsurgery (TEM) was introduced by Buess et al. ${ }^{11}$ Since its introduction, many surgical practices have adopted this technique as the standard therapy for large rectal adenomas. With TEM, the adenoma can be removed en bloc, by submucosal dissection or full-thickness rectal wall excision. TEM has been shown to be an efficacious and safe treatment, and superior to traditional transanal resection..$^{12}$

The endoscopic treatment of large rectal adenomas has also evolved. Improvements of imaging techniques and endoscopic instruments have led to advanced endoscopic resection methods like EMR. This technique allows piecemeal removal of large polyps through submucosal lifting and multiple snare resections.

Although selection bias inevitably exists in case series, published studies suggest that TEM and EMR have comparable recurrence rates. ${ }^{13}$ Most recurrences can be treated again without requiring radical surgery. The literature furthermore suggests that EMR is associated with fewer complications and reduced hospital admission, without requiring general anaesthesia. This may lead to differences in costs and quality of life. We designed a multicentre randomised non-inferiority trial to compare the cost-effectiveness and cost-utility of TEM and EMR for the resection of large rectal adenomas.

\section{PATIENTS AND METHODS Study design}

The TRansanal endoscopic microsurgery vs. ENDoscopic mucosal resection (TREND) Study was designed as a randomised, open-label, non-inferiority trial conducted at 17 Dutch centres and 1 Belgian centre. The non-inferiority design was chosen because the potential advantages of EMR from a patient and societal perspective like a lower complication rate, reduced hospital admission and absence of a need for general anaesthesia were considered irrelevant, unless EMR could be proven at least as effective as TEM in preventing recurrences. The study coordinator collected coded data at each study site. The study protocol was described previously. ${ }^{14}$

\section{Patients}

We recruited patients above 17 years of age, who had a large $(\geq 3 \mathrm{~cm})$, non-pedunculated rectal adenoma; at least $50 \%$ of the adenoma needed to be situated within $15 \mathrm{~cm}$ from the dentate line. All patients underwent white light endoscopy; adenomas were endoscopically assessed for any malignant features. In case of any suspicious features, endorectal ultrasound (EUS) was allowed to evaluate for deep submucosal invasion. EUS was not mandatory in the diagnostic workup. Biopsies of the lesion, if taken, did not show submucosal invasion at histopathological evaluation. Synchronous colonic lesions were excluded by complete colonoscopy. The general condition of patients permitted general and/or spinal anaesthesia (American Society of Anesthesiologists (ASA) classification 1-3). Every eligible patient was evaluated by a surgeon and a gastroenterologist, and both EMR and TEM were judged feasible. Patients with a suspicion of malignancy based on endoscopic features, biopsies or EUS, as well as patients with a life-threatening systemic disease or moribund clinical condition (ASA classification IV-V), a non-correctable coagulopathy or other contraindications for rectal surgery were excluded. All patients provided written informed consent.

\section{Randomisation}

Randomisation was done by the central study coordinator using a web-based randomisation module. Computer-generated block randomisation with a 1:1 allocation ratio and concealed random block sizes of two, four and six patients were used. Randomisation was stratified according to primary or recurrent nature of adenoma. Due to the invasive nature of the interventions and the logistics involved, neither the trial participants nor the investigators could be masked to group allocation.

\section{Intervention strategies}

TEM was performed as described by Buess. ${ }^{11}{ }^{14}$ The rectal defect was closed in the transverse direction. When TEM turned out to be technically impossible after randomisation, patients underwent subsequent EMR. EMR was performed as described by Karita and Hurlstone and argon plasma coagulation (APC) of the edges of the mucosal defect was prescribed in the protocol. ${ }^{14-16}$ When it turned out that EMR was technically not possible after randomisation or when EMR failed to remove $>90 \%$ of the adenoma, the patient subsequently underwent TEM. Standard discharge criteria were applied and included normal intake and mobility, and absence of fever.

In case of an unexpected submucosally invasive cancer despite adherence to the inclusion criteria, the patient was excluded after the histopathological evaluation (late exclusion).

After 3 months, follow-up endoscopy was performed for assessment of potential adenoma remnants. This procedure was performed by the treating endoscopist or, in patients who had undergone TEM, by the local endoscopist primarily involved in the study. Presumed residual disease was resected endoscopically and assessed histologically to confirm the presence of neoplasia. Residual disease at 3 months was not considered as recurrence; endoscopic intervention at 3 months was considered part of the primary treatment strategy.

\section{Follow-up}

All patients underwent surveillance endoscopies at 6 months, 12 months and 24 months after the primary treatment procedure by an independent endoscopist, who was blinded for the allocated study arm. Targeted biopsies of presumed recurrent adenoma were taken for histopathological confirmation. Random biopsies 
were taken from apparently normally healed scars to detect occult recurrence. Recurrence was defined as per Higaki criteria, as the presence of tumour at or nearby (within $5 \mathrm{~mm}$ ) the resection scar, or evidence of tumour with associated fold convergence. ${ }^{17}$ Follow-up via telephone was performed by research nurses after 1 day, 2 weeks, 3 months, 6 months, 12 months and 24 months.

\section{Outcomes}

The primary outcome was recurrence of neoplasia (adenoma remnants at 3 months were not considered as recurrence), and the number of days a patient was alive, outside the hospital and free of recurrence during the 2-year follow-up period. Secondary outcomes were complication rates, health-related and disease-specific quality of life as assessed by the EQ-5D-3L, SF-36, Wexner Continence Grading Scale and Colorectal Functional Outcome (COREFO) questionnaires, anorectal function and costs. ${ }^{18-21}$

A blinded expert panel of two gastroenterologists and two surgeons assessed the severity (according to Clavien-Dindo) of all adverse events that occurred during the study and their relatedness to the study procedure. ${ }^{22}$ Discrepancies were discussed until consensus was reached.

\section{Statistical analysis}

The principal analysis consisted of an intention-to-treat comparison of recurrence rates in the two treatment groups. The goal was to test for non-inferiority of EMR with respect to the primary outcome, and superiority with respect to secondary outcomes. Our previously published meta-analysis was used for sample size calculation. ${ }^{13}$ The margin of non-inferiority applied in the TREND Study was 6.7\%. It was assumed that the recurrence rate in the TEM group would be $3.3 \%$ and that EMR would be considered non-inferior if the recurrence percentage would remain below $10 \%$ at maximum. ${ }^{14}$ Assuming a baseline recurrence rate of $3.3 \%$ for both TEM and EMR, we would consider EMR to be non-inferior if the associated recurrence rate was less than 6.7 percentage points above the TEM recurrence percentage. We used a one-sided significance level of 0.05 . To attain a power of $80 \%, 89$ patients were needed in each group. The $\chi^{2}$ test was applied to compare recurrence rates. The number of days not spent in hospital was compared by the Wilcoxon rank-sum test. Quality of life questionnaires were analysed using linear mixed models. IBM SPSS Statistics V.22 was used for statistical analyses. The trial is registered in the Dutch Trial Registry, trial number NTR1422.

\section{Economic evaluation}

Cost-effectiveness (costs per recurrence-free patient) and costutility (costs per quality-adjusted life year (QALY)) were assessed from a societal perspective for a time-horizon of 24 months. The societal perspective included the costs of healthcare (index interventions, interventions after 3 months, hospital stay, major related diagnostic and therapeutic interventions, consultations with inhospital medical specialists, paramedics or alternative healers, formal home care), the costs of sick leave from work and non-reimbursable out-of-pocket expenses (over-the-counter medication, incontinence pads, additional private help).

\section{Quality and safety monitoring and interim analysis}

Dutch and Belgian gastroenterologists and surgeons were eligible for participation in the trial if they had performed at least 10 EMR or TEM procedures of (colo)rectal lesions larger than $3 \mathrm{~cm}$, respectively. Surgeons were required to have followed a formal training programme for TEM. Participating endoscopists mutually aligned EMR techniques after discussion in investigator meetings before including their first patient in the study. An expert panel evaluated a video-recorded procedure of each participating physician prior to commencement of the trial at that particular centre. All study procedures were monitored and video-recorded by a research nurse from the coordinating trial centre.

Serious adverse events (SAEs), according to the WHO definition, were reported to the institutional review board every 3 months. After 88 study patients had been treated, the suspicion of a potential uneven distribution of SAEs between both randomisation arms arose. At that time, safety and effectiveness were assessed in an interim analysis to reveal any potential imbalances between the two groups. An independent Data Safety Monitoring Board (consisting of a surgeon, a gastroenterologist and a clinical epidemiologist) was instituted, which recommended proceeding with the trial according to its original protocol.

\section{RESULTS}

From February 2009 until September 2013, 209 patients with large rectal adenomas were randomised (EMR 106; TEM 103). Two patients in both groups withdrew consent. One patient with prostate carcinoma instead of rectal adenoma in the EMR group was excluded. After the exclusion of these 5 patients, 103 patients in the EMR group and 101 patients in the TEM group were included in the analysis. In the EMR group, five patients underwent TEM because of EMR failure. In the TEM group, three patients underwent EMR because of TEM failure. Twenty-seven patients (13\%; EMR 15; TEM 12) had an unexpected malignancy and were excluded. One additional patient withdrew consent. Eventually, 176 patients were included in the long-term analyses (figure 1). Baseline characteristics of these patients were comparable (table 1). Characteristics of the 27 patients with unexpected rectal cancer (16 men, $68 \pm 9$ years old, 41\% ASA 1, $59 \%$ ASA 2) were also comparable. Their rectal lesions measured $47 \pm 12 \mathrm{~mm}$, and were situated $6.4 \pm 3.6 \mathrm{~cm}$ from the anal verge. Most (74\%) malignant lesions were sessile. Preoperative biopsies were taken in 26/27 patients, showing low-grade dysplasia in 19 and high-grade dysplasia in 7.

Most EMR procedures were performed under sedation $(77 \%$ conscious sedation, $17 \%$ deep sedation, $5 \%$ no sedation, $1 \%$ missing data). Submucosal lifting was scored as adequate in $90 \%$ of cases. APC was applied in 74 cases (84\%) after snare resection. In 76 cases (87\%), the EMR procedure was judged endoscopically radical by the endoscopist. Five patients (6\%) required an extended procedure (median of 2 EMR attempts, IQR 2-3). TEM was mostly performed under general anaesthesia (94\%; $6 \%$ spinal anaesthesia). A full thickness excision was done in 75 cases (84\%); 78 adenomas were excised as single piece (88\%). The peritoneal cavity was breeched in seven cases $(8 \%)$; two required conversion to transabdominal approach to close the defect. Histopathological evaluation of TEM specimens revealed complete margins (R0) in 59 cases (66\%); margins were incomplete (R1) in $14(16 \%)$ and unsure $(\mathrm{Rx})$ in 16 cases (18\%). Further procedure characteristics are summarised in table 2. Patients undergoing EMR were hospitalised significantly shorter than those undergoing TEM ( 0 days vs 1 day; $\mathrm{p}<0.001)$.

During repeat endoscopy at 3 months, there were three patients with adenoma remnants in the TEM group; they underwent snare resection $(n=2)$ or biopsy $(n=1)$ of the remnant. After EMR, there were three patients in whom adenoma remnants 


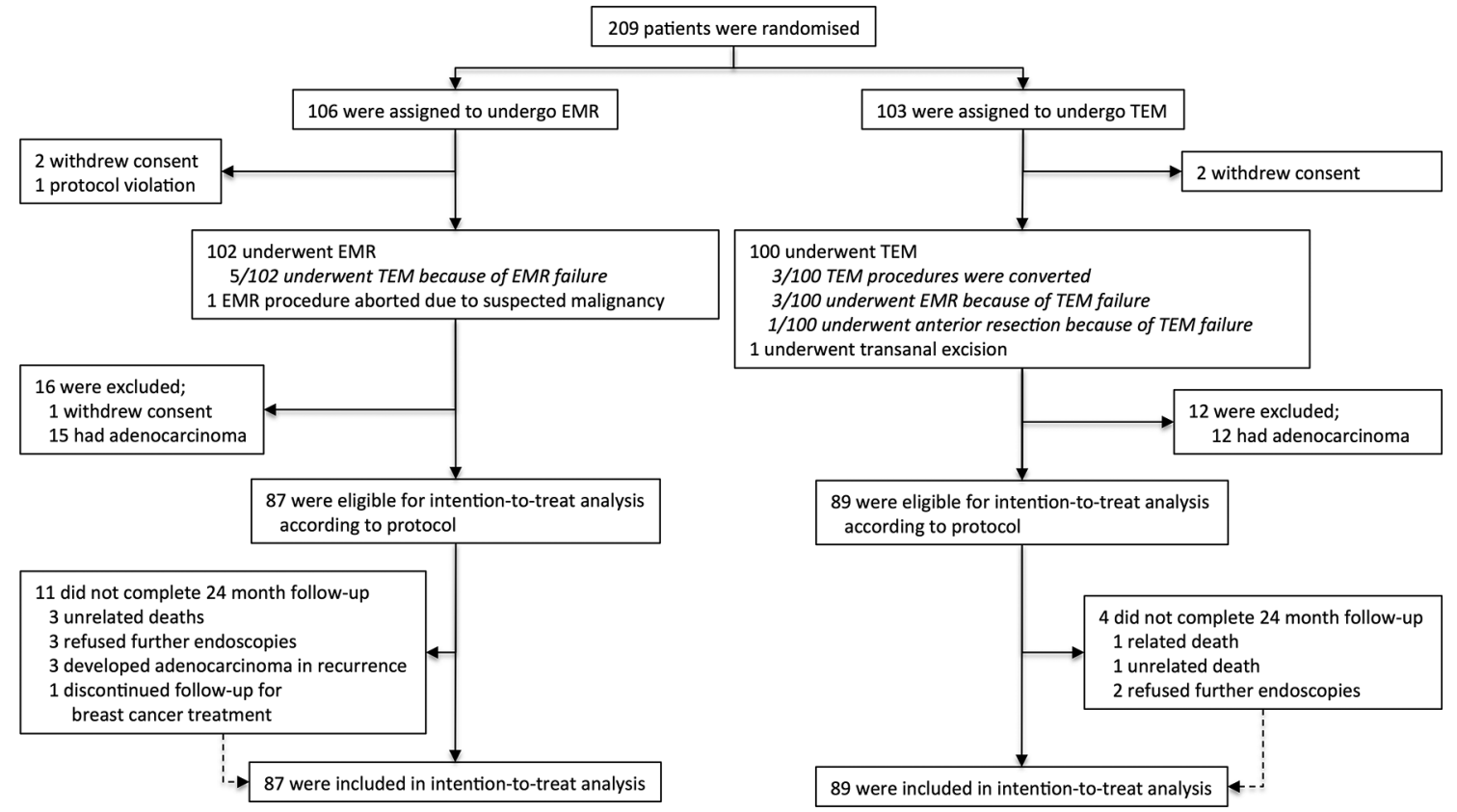

Figure 1 Enrolment and outcomes $190 \mathrm{~mm} \times 142 \mathrm{~mm}$ (300 × 300 DPI). EMR, endoscopic mucosal resection; TEM, transanal endoscopic microsurgery.

were discovered through random biopsies of a normally appearing scar. As such, they did not undergo any additional treatment. The 13 patients with visible remnants after EMR underwent snare resection $(\mathrm{n}=4)$, APC $(\mathrm{n}=1)$, a combination $(n=3)$ or a combination of cold biopsy and APC $(n=5)$. Endoscopic removal of adenoma remnants was required significantly more often after EMR (19\% vs 5\%; $\mathrm{p}=0.008)$.

The proportion of patients with recurrence was $15 \%$ in the EMR group and $11 \%$ in the TEM group (relative risk (RR) 1.33, one-sided $95 \%$ upper limit 2.46). This upper limit corresponds to an adenoma recurrence proportion of 0.276 . Hence, it cannot be ruled out that the absolute difference in adenoma recurrence proportions between EMR and TEM will exceed the non-inferiority margin of 0.067 (table 3), an inconclusive study result. ${ }^{23}$ The median time to recurrence was 7 months (IQR 6-12) after EMR and 12 months (IQR 7-21) after TEM ( $\mathrm{p}=0.10)$. In four patients who underwent EMR (5\%), occult recurrence was diagnosed from random biopsies in a normally healed scar. Of 87 patients who had undergone EMR, three developed adenocarcinoma in a recurrence. The mean number of days that patients spent out of the hospital while free of recurrence was comparable between groups (609 (95\% CI 557 to 654) in EMR, 652 (95\% CI 612 to 690$)$ in TEM, difference -44 (95\% CI -100 to -16$)$, $\mathrm{p}=0.15$ ). Subsequent sensitivity analyses, excluding any unrelated admissions, maintained comparable numbers.

Any postprocedural complication occurred in 16 patients after EMR (18\%) and 23 after TEM (26\%) (OR 0.65 (0.32-1.33), $p=0.23)$. Complications are further specified in table 3. Haemorrhage was the most common complication in both groups and repeat endoscopy was often performed. The Clavien-Dindo classification of postprocedural haemorrhage is specified in online supplementary table S1 (supplementary appendix). Major complications (Clavien-Dindo grade III-b and up) occurred in $1 \%$ (EMR) versus $8 \%$ (TEM) (OR 0.14 (95\% CI 0.02 to 1.13 ), $\mathrm{p}=0.064)$.

Anorectal manometry was performed before and 6 months after treatment in a subset of 24 study patients. Results of this study have been published before, demonstrating that anal sphincter pressure, anorectal reflexes, rectal sensation and compliance were not affected by adenoma resection. ${ }^{24}$ SF-36, EQ-5D-3L, Wexner and COREFO Scores are summarised in online supplementary tables S2-S6. Health related quality of life was comparable between groups. Patients undergoing TEM scored significantly less favourable on the Wexner Continence Grading Scale $(p=0.026)$, although continence improved after adenoma resection regardless of allocated treatment. COREFO Scores were significantly higher after TEM in comparison with $\mathrm{EMR}$, indicating worse function. Differences were more notable at short term.

Online supplementary table S7 shows the costs of inpatient and outpatient hospital resources, out-of-hospital care, informal care and out-of-pocket expenses. The mean total costs of the index interventions after summing the costs of EMR and TEM are $€ 777$ ( $€ 681$ to $€ 900$ ) in the EMR group and $€ 2177$ ( $€$ 2114 to $€ 2243)$ in the TEM group $(\mathrm{p}<0.001)$. There was a non-significant difference in costs $(-€ 1471$ ( $-€ 3428$ to $€$ 52)) of inpatient hospital days $(\mathrm{p}=0.115)$. The costs of major related procedures were comparable between groups. No differences in medical consultations, use of formal and informal home care, or out-of-pocket expenses were observed. The costs of lost working hours due to absence from work were comparable $(p=0.61)$. Table 4 shows the total and subtotal costs. EMR was associated with lower costs compared with TEM $(-€ 3003$ $(-€ 6353$ to $-€ 84)$ ). Based on the generic Dutch general population preferences, generated QALYs were comparable (EMR 1.73 (1.65-1.80), TEM 1.73 (1.64-1.81), $\mathrm{p}=0.96)$. Online supplementary figure $\mathrm{S} 1$ shows the cost-effectiveness plane of differences in costs versus differences in QALYs. The probability of EMR being cost-effective when compared with TEM is reflected in the 'cost-effectiveness acceptability curve' in online supplementary figure $\mathrm{S} 2$.

Tumour staging and additional treatment of the unexpected malignancies are shown in table 5. After a follow-up of more than 4 years, overall survival was $100 \%$. One locally recurrent rectal cancer was detected 22 months after TEM during a planned surveillance endoscopy. Distant metastases were found in three patients during 
Table 1 Clinical and pathological characteristics of included patients at baseline

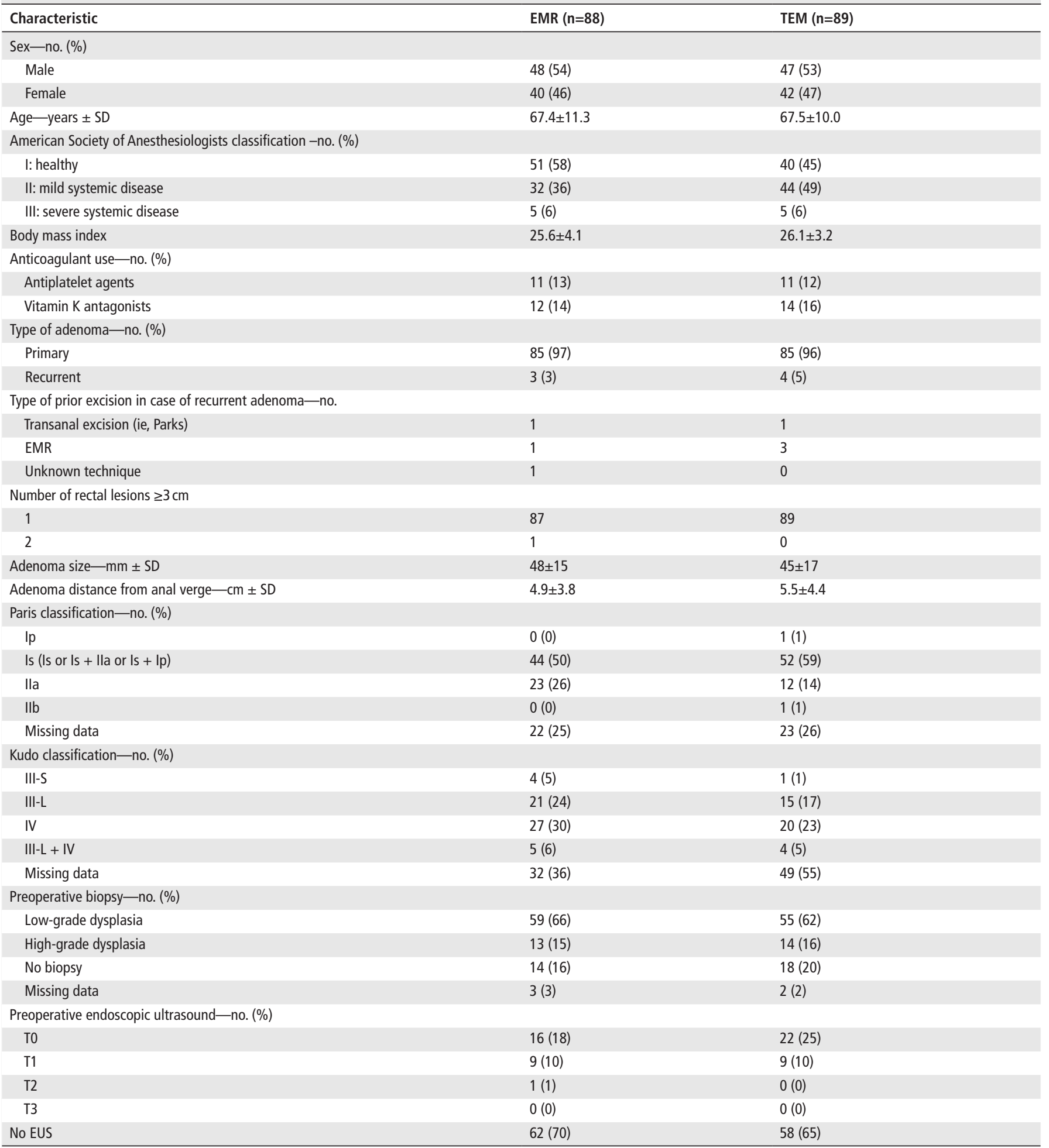

EMR, endoscopic mucosal resection; EUS, endorectal ultrasound; TEM, transanal endoscopic microsurgery.

surveillance after TEM of a low-risk T1 cancer (all R0 resections); these consisted of pulmonary metastases in two patients and a solitary liver metastasis in one. Three out of four patients with recurrent malignant disease or distant metastases were treated with curative intent with either additional surgery or radiotherapy.

\section{DISCUSSION}

In this randomised trial, EMR was evaluated as a non-inferior alternative for TEM for resection of large rectal adenomas. Due to unexpected high recurrence rates of both TEM and EMR, partly due to a small absolute difference in recurrence rates (4\%), non-inferiority of EMR could not be demonstrated. We found an early remnant rate of 19\% after EMR and 5\% after TEM, and a recurrence rate of $15 \%$ and $11 \%$, respectively. The number of days that patients spent out of the hospital while free of recurrence was comparable between groups.

With the point estimate for the EMR recurrence rate already above the one for TEM, we reported the one-sided 95\% upper 


\begin{tabular}{|c|c|c|c|}
\hline Characteristic & $\operatorname{EMR}(n=88)$ & TEM (89) & $p$ \\
\hline Median procedure time- -minutes (IQR) & $53(35-90)$ & $60(44-84)$ & 0.61 \\
\hline Median hospital stay—days (IQR) (range) & $0(0-1)(0-8)$ & $1(1-2)(0-84)$ & $<0.001$ \\
\hline Endoscopic completeness of resection-no. (\%) & $76(87)$ & $84(94)$ & 0.11 \\
\hline Extended procedure (multiple attempts required) & $5(6)$ & N/A & \\
\hline Median no. of attempts (IQR) & $2(2-3)$ & & \\
\hline Intraprocedural events—no. (\%) & & & 0.03 \\
\hline Haemorrhage & $2(2)$ & $2(2)$ & \\
\hline Peritoneal breech & $0(0)$ & $7(8)$ & \\
\hline Defect closed via TEM & N/A & 5 & \\
\hline Defect closed via transabdominal approach & $\mathrm{N} / \mathrm{A}$ & 2 & \\
\hline Mortality & $0(0)$ & $0(0)$ & \\
\hline Other & $2(2)$ & $0(0)$ & \\
\hline Procedure failure (\%) & $5(6)$ & $3(3)$ & 0.50 \\
\hline Subsequent TEM & 5 & N/A & \\
\hline Subsequent EMR & N/A & 2 & \\
\hline Subsequent TME & 0 & 1 & \\
\hline Subsequent oncological workup (suspected malignancy) & 0 & 0 & \\
\hline Histopathological classification—no. (\%) & & & 0.52 \\
\hline Dysplasia NOS & $2(2)$ & $5(6)$ & \\
\hline Low-grade dysplasia & $39(44)$ & $38(43)$ & \\
\hline High-grade dysplasia & $47(53)$ & $46(52)$ & \\
\hline \multicolumn{4}{|l|}{ Histopathological completeness of resection— no. (\%) } \\
\hline R0-radical & N/A & $59(66)$ & \\
\hline R1-irradical & N/A & $14(16)$ & \\
\hline $\mathrm{Rx}$-unsure & $88(100)$ & $16(18)$ & \\
\hline
\end{tabular}

EMR, endoscopic mucosal resection; TEM, transanal endoscopic microsurgery; TME, total mesorectal excision.

confidence limit in this non-inferiority study rather than its equivalent $90 \%$ two-sided CI.

The trial was conducted in 18 largely non-academic hospitals and in the absence of tertiary referral centres. EMR and TEM were performed by selected gastroenterologists and surgeons with a specific dedication to these procedures. In this setting, recurrence rates have turned out higher than anticipated. In a previous meta-analysis, we have shown that the effectiveness of EMR and TEM are similar, when allowing a second resection of adenoma remnants. ${ }^{13}$ These remnants can often be easily treated by repeat EMR or by minor interventions using simple snare resection, biopsy forceps or APC. Hypothesising that such early resection of adenoma remnants might reduce recurrence rates, any intervention at 3 months was part of the initial treatment strategy in our study protocol. Although the 3-month endoscopy was not performed in 22/89 TEM patients, this was the case in only $2 / 10$ patients with recurrence after TEM. In the EMR group, APC was omitted in $16 \%$ of patients $(n=12)$. However, recurrence was only observed in $2 / 12$. Considering the observed TEM recurrence rate (11\%), adhering to the proposed additional $6.7 \%$ for EMR would require over 270 patients per group to preserve a statistical power of $80 \%$. In contrast, proportional adjustment of allowed EMR recurrence rates would be clinically unacceptable.

A more recent EMR study and review (with different inclusion criteria) report late recurrence rates of $16 \%$ to $20 \%{ }^{25}{ }^{26} \mathrm{~A}$ large prospective EMR series in an highly specialised setting reports a late recurrence rate of only $4 \% .{ }^{27}$ However, this percentage is importantly flawed by excluding $16 \%$ of patients, with residual disease at their first follow-up endoscopy from further recurrence analyses. The available TEM series are mainly case mixes of benign and malignant rectal lesions. An Italian series of TEM for rectal adenomas reports a $5.6 \%$ recurrence rate. ${ }^{28}$

Economic evaluation showed that EMR is cheaper than TEM in $99 \%$ of cases, saving approximately $€ 3000$ per patient, whereas a difference in QALYs could not be demonstrated. Therefore, from a health economic perspective, EMR is the preferable treatment.

Both EMR and TEM were associated with significant postprocedural morbidity. Repeat endoscopy for haemorrhage was often performed, even though endoscopic intervention was not always necessary. Currently, many study groups employ more permissive definitions of clinically significant haemorrhage. Therefore, our numbers may be an overestimation. Although the distinction between major and minor complications was not significantly different between groups, higher patient numbers might have shown significance.

Self-reported functional outcome improved after adenoma resection regardless of allocated treatment. The differences in Wexner and COREFO Scores in favour of EMR, which were more notable at short term, may indicate a potential disadvantageous effect of the 4-cm TEM proctoscope or loss of compliance due to transmural resection, although this is not supported by the literature.

A generally acknowledged advantage of TEM over EMR is the possibility to perform a single-piece excision, enabling accurate histopathological assessment of resection margins. Unfortunately, the rates of Rx and R1 of TEM specimens were 18\% and $16 \%$, respectively. The cases of $\mathrm{Rx}$ may reflect unfamiliarity of local pathologists with TEM specimens; the absence of centralised histopathological evaluation is a limitation of our study. However, the $16 \%$ R1 ratio is considered substandard in comparison with the current literature. 


\begin{tabular}{|c|c|c|c|}
\hline Outcome & $\operatorname{EMR}(n=87)$ & TEM $(n=89)$ & $\mathrm{p}$ \\
\hline Follow-up endoscopy within 3 months-no. (\%) & $85(98)$ & $67(75)$ & $<0.001$ \\
\hline \multicolumn{4}{|l|}{ Surveillance endoscopy—no (\%) } \\
\hline 6 months & 82 & 78 & 0.13 \\
\hline 12 months & 81 & 81 & 0.61 \\
\hline 24 months & 79 & 85 & 0.22 \\
\hline Adenoma remnants at 3 months—no. (\%) & $16(19)$ & $3(5)$ & 0.008 \\
\hline Adenoma recurrence at 6 months, 12 months or 24 months-no. (\%) & $13(15)$ & $10(11)$ & $0.23^{*}$ \\
\hline \multicolumn{4}{|l|}{ Number of recurrences during study follow-up } \\
\hline 1 & 10 & 7 & \\
\hline 2 & 2 & 2 & \\
\hline 3 & 1 & 1 & \\
\hline Any adenoma remnant or recurrence during follow-up - no. (\%) & $23(26)$ & $11(12)$ & N/A \\
\hline Carcinoma in recurrence- - no. (\%) & $3(3)$ & $0(0)$ & 0.24 \\
\hline Median time to recurrence-months (IQR) & $6.8(6.1-12.2)$ & $12.0(6.8-20.7)$ & 0.10 \\
\hline Time alive, recurrence-free and out of hospital—days \pm SD & $609 \pm 209$ & $652 \pm 188$ & 0.16 \\
\hline Patients with complication—no. (\%) & $16(18)$ & $23(26)$ & 0.23 \\
\hline Patients with major complication—no (\%) & $1(1 \%)$ & $7(8 \%)$ & 0.064 \\
\hline \multicolumn{4}{|l|}{ No. of complications per patient } \\
\hline 1 & 12 & 20 & \\
\hline 2 & 3 & 1 & \\
\hline 3 & 1 & 2 & \\
\hline \multicolumn{4}{|l|}{ Complications -no. } \\
\hline Any & 21 & 28 & \\
\hline Hemorrhage & 19 & 15 & \\
\hline Suture dehiscence & 1 & & \\
\hline Perforation & & 1 & \\
\hline Rectal stenosis & & 4 & \\
\hline lleus / gastroparesis & & 1 & \\
\hline Myocardial infarction & & 1 & \\
\hline Respiratory failure & & 1 & \\
\hline Urinary tract infection & 1 & 3 & \\
\hline Other & & 2 & \\
\hline \multicolumn{4}{|l|}{ Clavien-Dindo classification—no. } \\
\hline I & 3 & 8 & \\
\hline II & 3 & 6 & \\
\hline III-a & 14 & 7 & \\
\hline III-b & 1 & 3 & \\
\hline IV & 0 & 3 & \\
\hline V & 0 & 1 & \\
\hline
\end{tabular}

${ }^{*}$ The null hypothesis that EMR would lead to an increase of at least 6.7 percentage points above the recurrence rate in the TEM group, is not rejected ( $p=0.23$, Farrington and Manning)

†Not assessed, because the actual hypothesis testing according to protocol included the superiority of TEM concerning residual disease (underpinning the need for a second EMR procedure at 3 months as part of the primary intervention) and the non-inferiority of EMR concerning recurrent disease.

$\ddagger$ Other complications: $1 \mathrm{x}$ thrombophlebitis, treated with oral antibiotics; $1 \mathrm{x}$ abdominal pain associated with free air in the retroperitoneum.

EMR, endoscopic mucosal resection; TEM, transanal endoscopic microsurgery.

\begin{tabular}{|c|c|c|}
\hline & $\begin{array}{l}\text { EMR }(n=87) \\
\text { Mean costs in } €(\mathrm{BCa} 95 \% \mathrm{Cl})^{*}\end{array}$ & $\begin{array}{l}\text { TEM }(n=89) \\
\text { Mean costs in } €(\mathrm{BCa} 95 \% \mathrm{Cl})^{*}\end{array}$ \\
\hline \multicolumn{3}{|l|}{ Resource } \\
\hline Healthcare & 3730 (2825 to 4729$)$ & 6668 (5136 to8629) \\
\hline Informal care and out-of-pocket & 420 (247 to631) & 791 (392 to1287) \\
\hline Production loss & 964 (276 to1838) & 659 (97 to1406) \\
\hline Total & 5115 (3876 to6532) & 8118 (6067 to10602) \\
\hline
\end{tabular}

* $\mathrm{BCa} 95 \% \mathrm{Cl}$ : bias corrected and accelerated $95 \% \mathrm{Cl}$.

EMR, endoscopic mucosal resection; TEM, transanal endoscopic microsurgery. 
Table 5 Staging and additional treatment of the unexpected rectal cancers

\begin{tabular}{|c|c|c|c|}
\hline & $\operatorname{EMR}(n=15)$ & TEM $(n=12)$ & $\mathrm{p}$ \\
\hline \multicolumn{3}{|l|}{ pT-stage-no. (\%) } & 0.33 \\
\hline $\mathrm{T} 1$ & $12(80)$ & $10(83)$ & \\
\hline $\mathrm{T} 2$ & $1(8)$ & $2(17)$ & \\
\hline $\mathrm{T} 3$ & $2(13)$ & - & \\
\hline \multicolumn{3}{|l|}{ Sm-stage-no. (\%) } & 0.55 \\
\hline Sm1 & $3(27)$ & $2(22)$ & \\
\hline Sm2 & $2(18)$ & $2(22)$ & \\
\hline Sm3 & $2(18)$ & $4(44)$ & \\
\hline Not assessable & $4(36)$ & $1(11)$ & \\
\hline \multicolumn{3}{|l|}{ pN-stage-no. (\%) } & 0.66 \\
\hline No & $5(33)$ & $5(42)$ & \\
\hline $\mathrm{Nx}$ & $10(67)$ & $7(58)$ & \\
\hline \multicolumn{4}{|l|}{ Differentiation grade-no. (\%) } \\
\hline Good-moderate & $10(83)$ & $8(73)$ & 0.55 \\
\hline Moderate & $2(17)$ & $2(18)$ & \\
\hline Mucinous & $0(0)$ & $1(9)$ & \\
\hline Angioinvasion一no. (\%) & $0(0)$ & $0(0)$ & N/A \\
\hline Lymph invasion-no. (\%) & $0(0)$ & $0(0)$ & $\mathrm{N} / \mathrm{A}$ \\
\hline \multicolumn{3}{|c|}{$\begin{array}{l}\text { Histopathological completeness of } \\
\text { resection-no. (\%) }\end{array}$} & 0.04 \\
\hline RO & $8(53)^{*}$ & $11(92) \dagger$ & \\
\hline $\mathrm{Rx}$ & $7(47)$ & $1(8)$ & \\
\hline \multicolumn{3}{|l|}{ Additional therapy—no. (\%) } & 0.04 \\
\hline Surveillance & $3(20)$ & $7(58)$ & \\
\hline Completion surgery & $12(80)$ & $5(42)$ & \\
\hline \multicolumn{3}{|c|}{ Type of completion surgery-no. (\%) } & $0.04^{*}$ \\
\hline Abdominoperineal resection & $2(17)$ & $4(80)$ & \\
\hline Low anterior resection & $4(33)$ & $1(20)$ & \\
\hline TEM & $6(50)$ & - & \\
\hline
\end{tabular}

*EMR: RO when the basal margins were free of malignancy.

TTEM: RO when the basal and lateral margins were free of malignancy.

‡Remained not significant after performing the Bonferroni correction.

EMR, endoscopic mucosal resection; TEM, transanal endoscopic microsurgery.

Endoscopic submucosal dissection (ESD) was developed in Japan to enable endoscopic en bloc resection even in large lesions. A meta-analysis comparing the results of ESD and EMR has shown promising rates of en bloc resection, radical resections and recurrence. These rates were achieved at the expense of longer procedure times and higher complication rates. ${ }^{29}$ The short-term and long-term cost-effectiveness of ESD and EMR in the distal colon are currently investigated in a randomised trial. ${ }^{30}$

An important finding was the high rate of unexpected malignancies in $13 \%$ of large non-pedunculated rectal polyps that endoscopically appeared benign. This seems to be comparable to the existing literature in which large non-pedunculated adenomas harbour invasive disease between $6.9 \%$ and $14 \% .^{31-34}$ The protocol predicted a 1.6\%-3\% cancer rate by strict adherence to inclusion criteria. Regrettably, the Paris classification was not reported in 4/27 patients (14\%) with malignancies and Kudo was not reported in 15 (54\%). Of malignant lesions, preoperative biopsies showed only low-grade dysplasia in $68 \%$. Assessing risk factors for malignancy in the diagnostic workup of large rectal lesions extends beyond the scope of this study. Although training of accurate optical diagnosis of large colorectal lesions among endoscopists is strongly recommended, our data reflect its difficulty and emphasise the issue of sampling error in these large lesions. By late exclusion of unexpected malignancies, the study protocol has turned out to be a limitation of sound intention-to-treat-analyses.

The majority of these unexpected cancers were low risk T1. The need for completion surgery was significantly higher after EMR, but the proportion of radical TME was comparable between EMR and TEM. There was a tendency towards a higher permanent stoma rate after initial TEM, but numbers are too small to draw any conclusion. Overall survival was $100 \%$ and three of four recurrences that were found during more than 4 years of follow-up were treated with curative intent.

In the EMR group, three patients were diagnosed with carcinoma in a recurrence. In the first two patients, the primary adenoma involved $95 \%$ of the rectal circumference. Histopathology of the numerous EMR pieces revealed high-grade dysplasia but no submucosal invasion. After 6 months, a rectal stenosis was seen, and biopsies revealed submucosally invasive cancer. In those cases, a sampling error of the pathological examination of the primary treatment may be the reason for missing the diagnosis earlier, as extensive lesions like these often lead to jars with more than 20 small pieces being sent to the pathologist. The third patient had a persisting, benign looking, fibrotic ulcer during surveillance endoscopies after EMR of an adenoma, demonstrating high-grade dysplasia. Repeated biopsies were benign. After 1 year, EUS showed local rectal wall thickening, 
and biopsies revealed adenocarcinoma. The latter patient may have been misdiagnosed prior to randomisation; however, the interval seems relatively long to support this theory.

Although the number of patients included per participating centre was low, this does not reflect the actual annual EMR volume of the centres. First, at least half of the patients that were invited to participate declined because of preference for one or the other procedure. Second, lesions below $3 \mathrm{~cm}$ or extending too high in the sigmoid or too close to the dentate line had to be excluded. Third, the volume of rectal EMRs is maximally 25\% of the total colonic EMRs.

Our data reflect daily practice in the Netherlands and Belgium. Although the participating centres are gradually acquiring a tertiary referral setting, they have much lower case-volumes compared with the few expert centres dominating the current literature. The high rates of unexpected malignancies as well as the rather high recurrence and complication rates stress the importance of centralisation of these technically demanding techniques. As advanced endoscopic and transanal surgical skills are difficult to measure objectively, protocolled prospective registration should be employed. Training programmes focusing on optical diagnosis during endoscopy and advanced endoscopic and surgical techniques are highly recommended.

In summary, under the statistical assumptions of this study non-inferiority could not be demonstrated. However, EMR may have potential due to a tendency of lower complication rates and a better cost-effectiveness ratio. The high rate of unexpected cancers should be dealt with in further studies.

\section{Author affiliations}

Department of Surgery, Academic Medical Centre, Amsterdam, Netherlands

${ }^{2}$ Surgery, IJsselland Hospital, Capelle a/d IJssel, the Netherlands

${ }^{3}$ Surgery, Máxima Medical Centre, Veldhoven, the Netherlands

${ }^{4}$ Surgery, Meander Medical Centre, Amersfoort, the Netherlands

${ }^{5} \mathrm{Gastroenterology,} \mathrm{Leiden} \mathrm{University} \mathrm{Medical} \mathrm{Centre,} \mathrm{Leiden,} \mathrm{the} \mathrm{Netherlands}$

${ }^{6}$ Surgery, Catharina Hospital, Eindhoven, the Netherlands

${ }^{7}$ Surgery, Medical Centre Leeuwarden, Leeuwarden, the Netherlands

${ }^{8}$ Gastroenterology, Onze Lieve Vrouwe Gasthuis, Amsterdam, the Netherlands

${ }^{9}$ Gastroenterology, Amphia Hospital, Breda, the Netherlands

${ }^{10}$ Surgery, Amphia Hospital, Breda, the Netherlands

${ }^{11}$ Gastroenterology, Catharina Hospital, Eindhoven, the Netherlands

${ }^{12}$ Gastroenterology, Meander Medical Centre, Amersfoort, the Netherlands

${ }^{13} \mathrm{G}$ astroenterology, St. Antonius Hospital, Nieuwegein, the Netherlands

${ }^{14} \mathrm{Clinical}$ Research Unit, Academic Medical Centre, Amsterdam, Netherlands

${ }^{15}$ Gastroenterology, Academic Medical Centre, Amsterdam, the Netherlands

Correction notice This article has been corrected since it published Online First. The acknowledgements and collaborators statements have been updated.

Acknowledgements The authors thank the research nurses involved for their outstanding work collecting and managing data, all medical and nursing staff in participating hospitals for their efforts, and all patients for participating in the trial.

Collaborators The authors thank Bart Bijnen, Dept of Surgery, Medical Cente Alkmaar, Alkmaar, The Netherlands; Raf Bisschops, Dept of Gastroenterology, University Hospital Leuven, Leuven, Belgium; Paul Davids, Dept of Surgery, Diakonessenhuis, Utrecht, The Netherlands; Annekatrien Depla, Dept of Gastroenterology, Slotervaart Hospital, Amsterdam, The Netherlands; Hendrik van Dullemen, Dept of Gastroenterology, University Medical CenterGroningen, Groningen, the Netherlands; Michael F Gerhards, Dept of Surgery, Onze Lieve Vrouwe Gasthuis, Amsterdam, The Netherlands; Martin Houben, Dept of Gastroenterology, Haga Teaching Hospital, the Hague, The Netherlands; Klaas vander Linde, Dept of Gastroenterology, Medical Center Leeuwarden, Leeuwarden, The Netherlands; Rosalie Mallant, Dept of Gastroenterology, Flevo Hospital, Almere, The Netherlands; Robin Timmer, Dept of Gastroenterology, St Antonius Hospital, Nieuwegein, The Netherlands; Ben Witteman, Dept of Gastroenterology, GelderseVallei Hospital, Ede, The Netherlands; Albert Wolthuis, Dept of Surgery, University Hospital Leuven, Leuven, Belgium.

Contributors Study design: FJCvdB, EJRdG, MGD, PF, WAB, ED. Data collection: RMB, GDM, FJCvdB. Data analysis: RMB, GDM, MGD. Data interpretation: RMB, GDM, EJRdG, FJCvdB, ECJC, PGD, JCH, IHJTdH, CH, JMJ, AWMvMdW, GPvdS, EJS, MPS, BLAMW, MGD, PF, WAB, ED. First draft of the manuscript: RMB, with input from
GDM, EJRdG, MGD, PF, WAB, ED. Critical revisal of the manuscript: GDM, EJRdG, FJCvdB, ECJC, PGD, JCH, IHJTdH, CH, JMJ, AWMvMdW, GPvdS, EJS, MPS, BLAMW, MGD, PF, WAB, ED. Final approval of the manuscript: RMB, GDM, EJRdG, FJCvdB, ECJC, PGD, JCH, IHJTdH, CH, JMJ, AWMvMdW, GPvdS, EJS, MPS, BLAMW, MGD, PF, $W A B, E D$.

Funding The trial was sponsored by the Netherlands Organization for Health Research and Development (ZonMw, file number 17092201), which did not have access to outcome data during the trial and did not participate in data analyses or the preparation of the manuscript. No endoscopic or surgical equipment was donated by the manufacturer.

Competing interests Dr. Dijkgraaf reports grants from The Netherlands Organisation for Health Research and Development, during the conduct of the study. Dr. Fockens reports personal fees from Covidien, personal fees from Fujifilm, personal fees from Olympus, outside the submitted work. Dr. Jansen reports personal fees from MSD, personal fees from Abbvie, personal fees from Takeda and personal fees from Hospira, outside the submitted work. Dr. Schoon reports personal fees from Boston-Scientific, personal fees from Medtronic, personal fees from Olympus, outside the submitted work. Furthermore, we declare no competing interests.

Patient consent Detail has been removed from these case descriptions to ensure anonymity. The editors and reviewers have seen the detailed information available and are satisfied that the information backs up the case the authors are making.

Ethics approval Institutional Review Board at each study centre.

Provenance and peer review Not commissioned; externally peer reviewed.

(c) Article author(s) (or their employer(s) unless otherwise stated in the text of the article) 2018. All rights reserved. No commercial use is permitted unless otherwise expressly granted.

\section{REFERENCES}

1 Netherlands Cancer Registry, 2016. www.cijfersoverkanker.n

2 Regula J, Rupinski M, Kraszewska E, et al. Colonoscopy in colorectal-cancer screening for detection of advanced neoplasia. N Eng/ J Med 2006;355:1863-72.

3 Jacobs ET, Martínez ME, Alberts DS, et al. Association between body size and colorectal adenoma recurrence. Clin Gastroenterol Hepatol 2007:5:982-90.

4 Rajendra S, Ho JJ, Arokiasamy J. Risk of colorectal adenomas in a multiethnic asian patient population: race does not matter. J Gastroenterol Hepatol 2005;20:51-5.

5 Muto T, Nagawa H, Watanabe T, et al. Colorectal carcinogenesis: historical review. Dis Colon Rectum 1997:40:580-5.

6 Allen J. Molecular biology of Colon polyps and Colon cancer. Semin Surg Oncol 1995;11:399-405.

7 Winawer SJ, Zauber AG, Ho MN, et al. Prevention of colorectal Cancer by colonoscopic polypectomy. the National Polyp Study Workgroup. N Engl J Med 1993;329:1977-81.

8 Zauber AG, Winawer SJ, O'Brien MJ, et al. Colonoscopic polypectomy and long-term prevention of colorectal-cancer deaths. N Engl J Med 2012;366:687-96.

9 Moore J S, Cataldo P A, Osler T, et al. Transanal endoscopic microsurgery is more effective than traditional transanal excision for resection of rectal masses. Dis Colon Rectum 2008:51:1026-31.

10 Winde G, Nottberg H, Keller R, et al. Surgical cure for early rectal carcinomas (T1). Transanal endoscopic microsurgery vs. anterior resection. Dis Colon Rectum 1996:39:969-76.

11 Buess G, Hutterer F, Theiss J, et al. [A system for a transanal endoscopic rectum operation]. Chirurg 1984;55:677-80.

12 Lin GL, Meng WC, Lau PY, et al. Local resection for early rectal tumours: comparative study of transanal endoscopic microsurgery (TEM) versus posterior trans-sphincteric approach (Mason's operation). Asian J Surg 2006;29:227-32.

13 Barendse RM, van den Broek FJ, Dekker E, et al. Systematic review of endoscopic mucosal resection versus transanal endoscopic microsurgery for large rectal adenomas. Endoscopy 2011;43:941-9.

14 van den Broek FJ, de Graaf EJ, Dijkgraaf MG, et al. Transanal endoscopic microsurgery versus endoscopic mucosal resection for large rectal adenomas (TREND-study). BMC Surg 2009;9:4

15 Karita M, Tada M, Okita K, et al. Endoscopic therapy for early Colon cancer: the strip biopsy resection technique. Gastrointest Endosc 1991;37:128-32.

16 Hurlstone DP, Sanders DS, Cross SS, et al. A prospective analysis of extended endoscopic mucosal resection for large rectal villous adenomas: an alternative technique to transanal endoscopic microsurgery. Colorectal Dis 2005;7:339-44.

17 Higaki S, Hashimoto S, Harada K, et al. Long-term follow-up of large flat colorectal tumors resected endoscopically. Endoscopy 2003;35:845-9.

18 Ware JE. SF-36 Physical and Mental Health Summary Scales: a User's Manual. Boston, Mass: The Health Institute, New England Medical Center, 1994.

19 Sullivan M, Karlsson J, Ware JE. The Swedish SF-36 Health Survey--I. Evaluation of data quality, scaling assumptions, reliability and construct validity across general populations in Sweden. Soc Sci Med 1995;41:1349-58.

20 Jorge JM, Wexner SD. Etiology and management of fecal incontinence. Dis Colon Rectum 1993;36:77-97. 
21 Bakx R, Sprangers MA, Oort FJ, et al. Development and validation of a colorectal functional outcome questionnaire. Int J Colorectal Dis 2005;20:126-36.

22 Dindo D, Demartines N, Clavien PA. Classification of surgical complications: a new proposal with evaluation in a cohort of 6336 patients and results of a survey. Ann Surg 2004;240:205-13.

23 Piaggio G, Elbourne DR, Pocock SJ, et al. Reporting of noninferiority and equivalence randomized trials: extension of the CONSORT 2010 statement. JAMA 2012:308:2594-604

24 Barendse RM, Oors JM, de Graaf EJ, et al. The effect of endoscopic mucosal resection and transanal endoscopic microsurgery on anorectal function. Colorectal Dis 2013:15:e534-41.

25 Belderbos TD, Leenders M, Moons LM, et al. Local recurrence after endoscopic mucosal resection of nonpedunculated colorectal lesions: systematic review and meta-analysis. Endoscopy 2014;46:388-402.

26 Knabe M, Pohl J, Gerges C, et al. Standardized long-term follow-up after endoscopic resection of large, nonpedunculated colorectal lesions: a prospective two-center study. Am J Gastroenterol 2014;109:183-9.

27 Moss A, Williams SJ, Hourigan LF, et al. Long-term adenoma recurrence following wide-field endoscopic mucosal resection (WF-EMR) for advanced colonic mucosa neoplasia is infrequent: results and risk factors in 1000 cases from the australian colonic EMR (ACE) study. Gut 2015;64:57-65.
28 Allaix ME, Arezzo A, Cassoni P, et al. Recurrence after transanal endoscopic microsurgery for large rectal adenomas. Surg Endosc 2012;26:2594-600.

29 Fujiya M, Tanaka K, Dokoshi T, et al. Efficacy and adverse events of EMR and endoscopic submucosal dissection for the treatment of Colon neoplasms: a meta-analysis of studies comparing EMR and endoscopic submucosal dissection. Gastrointest Endosc 2015;81:583-95.

30 Backes Y, Moons LM, van Bergeijk JD, et al. Endoscopic mucosal resection (EMR) versus endoscopic submucosal dissection (ESD) for resection of large distal nonpedunculated colorectal adenomas (MATILDA-trial): rationale and design of a multicenter randomized clinical trial. BMC Gastroenterol 2016;16:56.

31 Moss A, Bourke MJ, Williams SJ, et al. Endoscopic mucosal resection outcomes and prediction of submucosal Cancer from advanced colonic mucosal neoplasia. Gastroenterology 2011;140:1909-18.

32 Kudo S, Lambert R, Allen JI, et al. Nonpolypoid neoplastic lesions of the colorecta mucosa. Gastrointest Endosc 2008:68:S3-S47.

33 Ramirez JM, Aguilella V, Gracia JA, et al. Local full-thickness excision as first line treatment for sessile rectal adenomas: long-term results. Ann Surg 2009:249:225-8.

34 Guerrieri M, Baldarelli M, de Sanctis A, et al. Treatment of rectal adenomas by transanal endoscopic microsurgery: 15 years' experience. Surg Endosc 2010;24:445-9. 